\title{
A model to improve the routing performance of Cognitive Radio Wireless Mesh Networks
}

\author{
Lesiba Morries Kola, Mthulisi Velempini \\ Department of Computer Science, University of Limpopo, Sovenga, South Africa
}

\begin{abstract}
The increasing demand for broadband wireless technologies has led to scarcity, inefficient utilisation, and underutilisation of the spectrum. The Cognitive Radio (CR) technology has emerged as the promising solution which improves the utilisation of the spectrum. However, routing is a challenge due to the dynamic nature of the CR networks. The link quality varies in space and time as nodes join and leave the network. The network connectivity is intermittent due to node mobility and the activities of the primary user. Spectrum aware, spectrum agile, and interference aware routing protocols are vital for the sturdiness of the network and efficient utilisation of the resources. We propose an interference aware, spectrum aware, and agile extended Weighted Cumulative Expected Transmission Time (xWCETT) routing protocol. The protocol integrates the features of the Ad-hoc On-demand Distance Vector (AODV) and the weighted cumulative expected transmission time (WCETT) routing protocols. The xWCETT was simulated using Network Simulator 2 and its performance compared with the AODV and the WCETT routing protocols. The xWCETT was evaluated with respect to quality of service related metrics and the results show that it outperformed the AODV and WCETT routing protocols.
\end{abstract}

Keywords: Cognitive radio, cognitive radio network, radio frequency spectrum, routing protocol, wireless mesh networks

Categories: • Networks $\sim$ Routing protocols $\bullet$ Networks $\sim$ Wireless mesh networks $\bullet$ Networks $\sim$ Network mobility $\bullet$ Networks $\sim$ Mobile networks $\bullet$ Networks $\sim$ Wireless local area networks $\bullet$ Networks $\sim$ Mobile ad hoc networks

Email:

Lesiba Morries Kola lesiba.kola@ul.ac.za (CoRreSPONDING), Mthulisi Velempini mthulisi.velempini@ul.ac.za
Article history:

Received: 25 Jul 2017

Accepted: 28 Sep 2017

Available online: 8 Dec 2017

\section{INTRODUCTION}

In the past decade, Wireless Mesh Networks (WMNs) (Akyildiz, Wang, \& Wang, 2005) have been recognised as a viable and cost effective technology that can extend wireless communication and broadband services to rural and remote areas. WMN technology has gained increased popularity because of its flexible architecture and its capability to provide integrated communication services without the need for wired infrastructure. A WMN is a wireless network formed by self-organizing, self-healing, and self-configuring nodes that are interconnected by wireless links to form a multi-hop mesh topology (Bruno, Conti, \& Gregori, 2005; Lee, Zheng, Ko, \& Shrestha, 2006a; Pabst et al., 2004). WMNs have benefits such as low deployment costs, simple network maintenance, network robustness, and extended service coverage. WMN technology integrates seamlessly with different

Kola, L.M. and Velempini, M. (2017). A model to improve the routing performance of Cognitive Radio Wireless Mesh Networks. South African Computer Journal 29(3), 64-80. https://doi.org/10.18489/sacj.v29i3.524

Copyright (C) the author(s); published under a Creative Commons NonCommercial 4.0 License (CC BY-NC 4.0).

$S A C J$ is a publication of the South African Institute of Computer Scientists and Information Technologists. ISSN 1015-7999 (print) ISSN 2313-7835 (online). 
wireless technologies such as the Institute of Electrical and Electronics Engineers (IEEE) 802.11, IEEE 802.15, IEEE802.16, and cellular network technology, as well as wireless sensor networks. However, WMNs operate in the Industrial Scientific and Medical (ISM) spectrum band which is the portion of radio frequency (RF) spectrum which is available for use without the requirements of a licence (Ho, Tweed, \& Le-Ngoc, 2017; Lee, Zheng, Ko, \& Shrestha, 2006b). It is worth noting that all the unlicensed portions of the ISM spectrum band are heavily utilised and overcrowded, especially in highly populated urban settings, and this results in very noisy channels, high levels of interference, and limited availability of bandwidth (Cheng, Jiang, \& Zhuang, 2006; Siraj \& Bakar, 2012).

The RF spectrum and bandwidth constraints suffered by traditional WMNs and other wireless network technologies operating in the unlicensed ISM spectrum bands significantly affect the overall performance of these networks, which is a challenge in meeting the requirements of real-time and quality of service (QoS) sensitive applications such as online gaming, voice over Internet protocol (VoIP), multimedia conferencing, and Internet protocol television (IPTV). To support these types of applications, new technological developments are required to meet the current and future demands for additional RF spectrum as more wireless technologies and applications are developed. The RF spectrum is a finite and very costly natural resource. Cognitive Radio (CR) technology (Siraj \& Bakar, 2012) has the potential to providing a novel wireless communication solution for the next-generation of wireless applications. CR technology refers a fully programmable system of wireless devices that are capable of sensing their operating environment and intelligently adapting their transmission parameters to provide excellent connectivity and communication (Haykin, 2005; Mitola, 1999).

CR technology aims to optimise the RF spectrum utilisation by allowing the unlicensed secondary users (SUs) to sense the RF spectrum and dynamically access the licensed portions of the RF spectrum bands on the condition that licensed primary users (PUs) are not interfered with (Akyildiz, Lee, Vuran, \& Mohanty, 2006; Akyildiz, Lo, \& Balakrishnan, 2011). Hence, integrating the traditional WMNs with CR technology promises to yield substantial benefits (Ishibashi \& Boutaba, 2009; Mitola, 2009), resulting in efficient bandwidth utilisation, increased network capacity and the overall improved network resource utilisation. Thus, CR technology is one of the key technologies to eliminate the challenges of underutilisation of expensive licensed RF spectrum resource, as well as overutilisation of the unlicensed RF spectrum bands.

On the other hand, integration of the CR technology with the traditional WMNs will offer other benefits, especially in the highly dense urban areas where the wireless systems operating in unlicensed spectrum bands are over-crowded. The multi-hop nature inherited from the WMNs will also offer wider coverage with shorter hops which means that data transmissions can occur with much lower transmission power. The resulting integrated structure is referred to as the next-generation of intelligent, frequency-shifting, and autonomous Cognitive Radio Wireless Mesh Network (CR-WMNs) (Akyildiz, Lee, \& Chowdhury, 2009). Figure 1 shows the design and the architecture of the CR-WMNs.

As illustrated in Figure 1, the architecture of CR-WMNs is formed by the two primary networks, i.e. the PU network as well as the SU network. The CR-WMNs use multiple spectrum bands for communication, i.e. different heterogeneous network technologies are able to coexist in time, frequency, and space. The PU network is made up of both the fixed base station and mobile nodes which communicate in the dedicated licensed bands. The PU has priority over all the channels in a given 


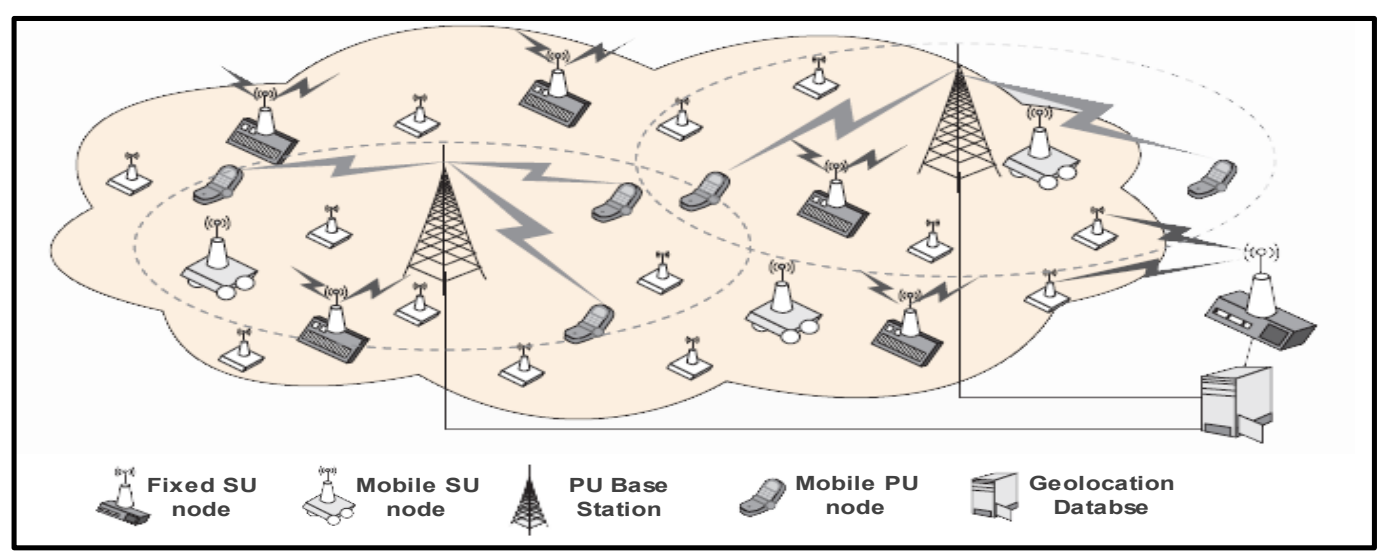

Figure 1: The architecture of CR-WMNs

band. The SU network is made up of fixed and mobile nodes which communicate opportunistically in both the licenced and unlicensed spectrum bands when the PU bands are not in use. The traditional WMNs are characterised by static to non-static topologies due to the presence of mobile SU nodes while the CRNs are characterised by spectrum agility (Sutton, Doyle, \& Nolan, 2006), spectrum awareness (Ji \& Liu, 2007; Noam, 1995), heterogeneity, and interoperability (Bhatia \& Li, 2007; Ramanathan, 2001; Zhang \& Tian, 2007; Tian \& Giannakis, 2006) as well as cognition (Ji \& Liu, 2007; Sengupta \& Subbalakshmi, 2013).

Although CR-WMNs have the potential to offer extended coverage, efficient spectrum resource utilisation, increased throughput, and improved network performance, CR-WMNs have to address the following challenges (Mitola, 1999; Akyildiz et al., 2006; Sutton et al., 2006; Ji \& Liu, 2007; Noam, 1995; Sengupta \& Subbalakshmi, 2013): dynamic topology due to non-static nodes, dynamic spectrum bands, heterogeneity in terms of standards and technologies, and the dynamic activities of licensed PUs. As a result, routing in such an environment is complex and challenging because more dynamic parameters must be taken into account when establishing the routes. Effective communication in any multi-hop wireless networks depends on a sustained connectivity amongst network nodes. The robustness and reliability of connectivity depends on the efficiency and effectiveness of the routing strategy employed in the network. As a result, the design, implementation and optimisation of an efficient routing protocol remain a critical part of a CR-WMN.

This paper proposes a spectrum aware, spectrum agile, and interference aware routing protocol designed for multi-radio and multi-channel CR-WMNs. The proposed routing protocol is referred to as the extended Weighted Cumulative Expected Transmission Time (xWCETT) routing protocol. The design, architecture, and functionality of the proposed xWCETT routing protocol is described in Section 3. 


\section{RELATED WORK}

Most recent literature in multi-hop wireless networks routing has focused on the traditional mobile ad-hoc networks, as well as cognitive radio ad-hoc networks (CRAHNs). However, there are a number of issues (Sengupta \& Subbalakshmi, 2013) that still require attention in multi-hop CRWMNs routing. Related work in CRAHNs focused on routing techniques such as joint routing and channel switching (Meghanathan \& Fanuel, 2015), joint next-hop and channel selection routing, interference-aware routing (Doomari \& Mirjalily, 2016), stability-based spectrum-aware routing (An, 2014), and location-aided spectrum-aware routing (Yadav \& Mane, 2015). However, our work focuses on path selection, spectrum-awareness, and spectrum-mobility in the presence of PU.

In Cacciapuoti, Calcagno, Caleffi, and Paura (2010), the authors proposed a reactive Cognitive Ad-hoc on-demand Distance Vector (CAODV) protocol for CRAHN environment. Their work focused on the design of routing protocol based on three optimisation criteria: avoiding the regions of PU activities, joint path, and channel selection at each forwarding node, and exploitation of multiple available channels to improve the network performance. However, the proposed CAODV protocol is based on a common control channel (CCC) strategy which is a challenge in the dynamic multi-hop CRN environment. The assumption of having the CCC may not be feasible due to the dynamic channel availability.

Sun, Zhang, Xie, Li, and Xu (2012) proposed a routing scheme that computes source-destination path by considering the activities of the PUs. The proposed algorithm makes use of the possibility of exploiting multiple frequencies at the same time between two SU nodes. The proposed scheme is based on joint routing and spectrum selection criteria that compute the most likely path that satisfies the requirements stated by the application. However, it implements concurrent multiple channel broadcast strategy which creates an overhead on all available channels, resulting in inefficient utilisation of the bandwidth.

Che-Aron, Abdalla, Abdullah, Hassan, and Rahman (2015) proposed a routing protocol for CRAHNs called the Robustness Aware Cognitive Ad-hoc Routing Protocol (RACARP). The proposed RACARP protocol's goal is primarily to provide the robust transmission path in the presence of PUs while offering the fast route recovery. The proposed protocol uses the Expected Path Delay (EPD) as a routing metric for path decision and fast route recovery. The protocol was implemented using the Network Simulator 2 (NS-2) and its performance was compared with the Dual Diversity Cognitive Ad-hoc Routing Protocol (D2CARP) which was proposed by Rahman, Caleffi, and Paura (2012). The RACARP protocol performed better than the D2CARP protocol in terms of the average throughput, packet loss rate, average end-to-end delay, and average jitter. The RACARP and the D2CARP protocols are able to adapt to dynamic PU activities however, the D2CARP employs the hop count routing metric which does not take into account the quality of links. As a result, inefficient hop count routing metric may frequently cause the D2CARP to establish non-optimal transmission paths thereby significantly degrading the performance of the protocol.

Another protocol named the E-D2CARP was proposed by Che-aron, Abdalla, Hassan, Abdullah, and Rahman (2014) as an improvement of D2CARP protocol. The E-D2CARP uses the Expected Path Delay (EPD) routing metric to make path decisions. During the route establishment process, the 
protocol uses the joint path and spectrum diversity and avoids the PU regions in order to ensure non-interference to PU activities. The protocol also ensures that it doesn't affect the PU activities in cases of path failure during route recovery process. The E-D2CARP protocol was also implemented in NS-2 and its performance was reported to be better that of its predecessor, the D2CARP protocol. This paper proposes a novel routing protocol that is based on efficient path, channel selection, spectrum-awareness, and spectrum-agility.

\section{THE XWCETT ROUTING PROTOCOL}

We investigated a number of routing protocols and routing metrics proposed for both the traditional WMN and CRN environment, how they were designed and how they perform under different scenarios. The outcome of our investigation is a proposed routing protocol called the xWCETT. The proposed routing protocol considers the merits of the popular AODV routing protocol as well as the merits of the multi-radio based WCETT. It combines the benefits of the AODV protocol with WCETT to form a new enhanced routing protocol. The primary rationale behind our proposed routing protocol is to construct an array of measured network metrics such as the throughput of each link, the end-to-end, latency, the stability of each link, the usage patterns of each channel per link, as well as the activities of the PU. The proposed xWCETT protocol therefore determines the bandwidth requirements, assesses the quality of each link, analyses the state of each channel, measures the activities of the PU and integrates the data transmission rates.

The proposed xWCETT routing protocol is based on a distributed local spectrum knowledge whereby each node in the network is responsible for constructing information about its immediate neighbours. The local knowledge information is shared amongst the neighbouring nodes through the common control channel (CCC). We assumed that all the nodes are tuned to the CCC to avoid broadcasting the control packets through all the channels, which reduces routing overhead. The route request (RREQ) and route reply (RREP) packets are sent through the CCC. The source node generates a RREQ message and forwards it to its neighbouring nodes through the CCC. The nodes receiving the RREQ packet process the message by checking if the message's destination IP address matches their own. If the node receiving the RREQ packet is not the destination node, it rebroadcasts the message. If the RREQ message's IP address matches, the receiving node generates the unicast RREP packet and sends it back to the source node through the path traversed by the RREQ packet. Upon receiving the RREP packet, the source node then transmits data in the best available channel. The records in the channel availability table are sorted or ranked according to the usage patterns. The best available channels are placed at the top of the channel availability table. The first channel in the table is then selected for data transmission. All the intermediate nodes follow the same process of selecting the best channel in the channel availability table to forward the data packets.

To compute the optimal source-destination path, the xWCETT routing protocol implements the three components, namely: the expected transmission count (ETX), the expected transmission time (ETT), and the Weighted Cumulative Expected Transmission Time (WCETT) routing metrics. The ETX measures the packet loss rate by estimating the number of MAC layer transmissions expected to successfully transmit the packet from source to the destination node. The ETT routing 
metric integrates the link transmission rates and the path cost calculation in order to improve the performance of the ETX metric. The third component of our routing protocol is the WCETT metric which is formulated by combining the two terms that are considered as trade-off between the end-toend latency as well as the throughput. We introduced the channel occupancy probability variable, $P_{c}$, which provides the channel availability estimate for all the channels on each link. The $P_{c}$ (as depicted in equation 1) computes the probability that a channel is unavailable for the SUs due the activities of the PU. The resulting routing metric for our proposed protocol is provided by Equation 1:

$$
\text { xwcett }_{m}=(1-\alpha) \sum_{i=1}^{n} \text { ETTl }_{i}+\left[\left(1-P_{c}\right) \alpha_{1 \geq c \geq C} \max _{c}\right]
$$

The $P_{c}$ represents the probability that the channel $c$ in a given $C$ set of channels is unavailable. We assume that each SU node is able to monitor and calculate the probabilistic availability measure of a channel based on the local knowledge of PU channel usage statistics. In the real scenario, this assumption would mean that each node will share its knowledge about the spectrum environment with its neighbours. As the nodes share their spectrum information, a global knowledge about availability of spectrum channels by all the nodes in the network is a possibility. Give the PU channel usage statistics derived from the channel availability table, this routing metric prioritizes stable source-destination routes by avoiding channels with higher probabilities of being occupied by the PUs.

The xWCETT routing protocol implements the xwcett_metric (depicted in Equation 1) to select the best routes. Our system model employs two radios where one radio monitors the availability of channels and the second radio for data transmission. At any point in time, each SU node monitors the PU activities on a set of allocated channels and associates appropriate probability values for each channel based on PU activities. In each case during the route establishment process, a RREP packet is generated based on the routing metric in Equation 1. The lowest metric value is used to determine the best route and therefore, data packets are transmitted on the selected path. The design architecture implemented in the SU network is illustrated in Figure 2.

The xWCETT routing protocol was implemented based on the multi-radio multi-channel architecture. The TCL script was used to configure the number of radios and channels required for the simulations. In our study, the number of radios was set to two (2) and the number of channels assigned to each radio was set to four (4) to closely emulate the ideal functionality of the CR-WMNs.

\section{METHODOLOGY}

The simulations were conducted using the open source object-oriented discrete-event NS-2 simulator, configured to run on the Ubuntu 12.04 Linux distribution. The cognitive radio cognitive network (CRCN) patch, which enables the cognitive radio capabilities in NS-2, was installed. Utilities such as the AWK programming language, perl scripting, python scripting, and Gnuplot were used for data manipulation, analysis, and graphical representation of the results.

We conducted a series of simulations based in varied network sizes ranging from 20 to 100 


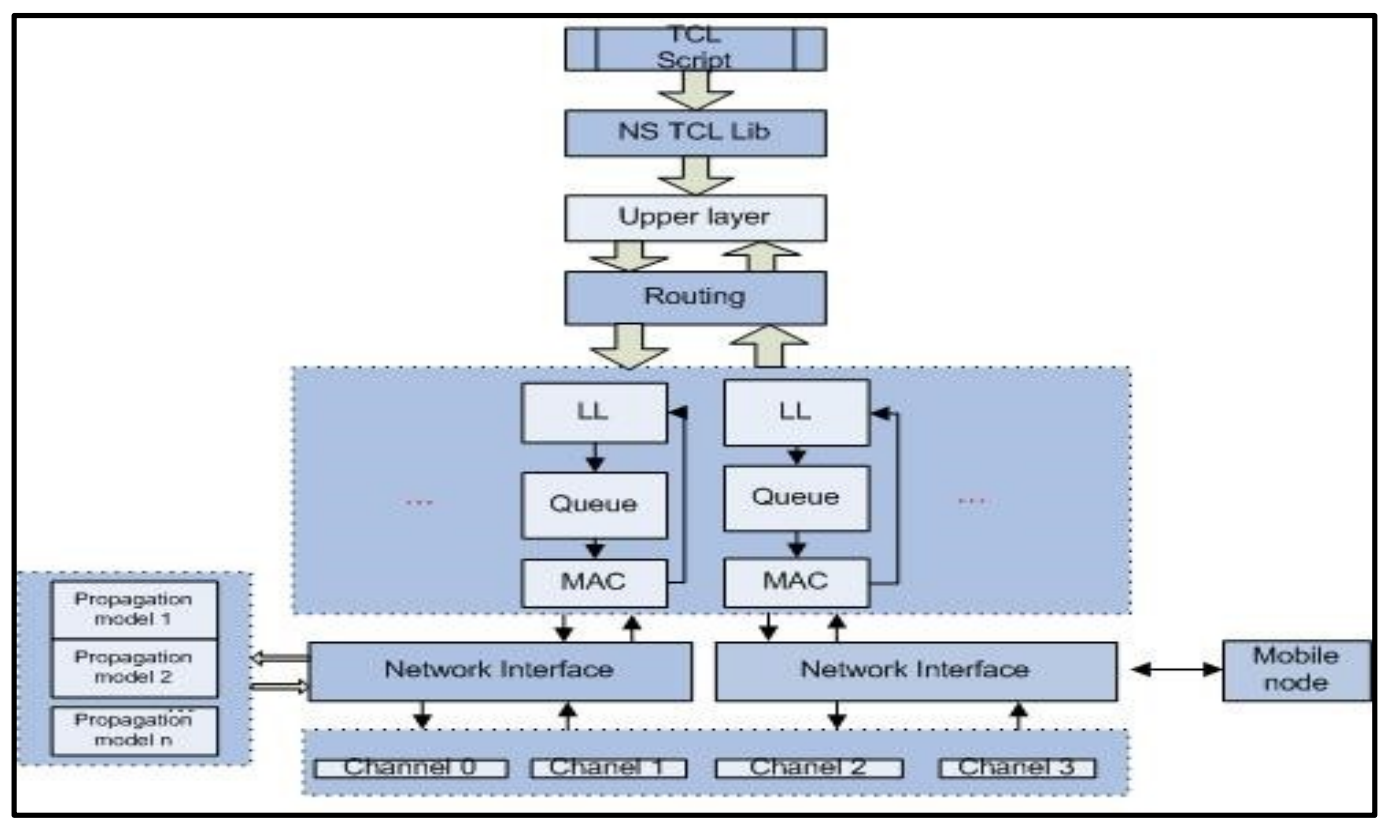

Figure 2: The design architecture of SU network (Fall \& Varadhan, 2007)

nodes. We evaluated the performance of AODV and CRCN-WCETT routing protocols and compared their performance with the xWCETT routing protocol. The simulations were run for 300 simulation seconds for each protocol. The simulation environment and parameters were configured according to Table 1. In each of the simulation scenarios conducted, we monitored and recorded the results of each scenario and how each routing protocol performed. The results are discussed in Section 5.

\section{RESULTS AND DISCUSSION}

Experiments were conducted in varied network sizes. In total, five (5) scenarios were considered to investigate how the network size affects the performance of the xWCETT routing protocol and to examine the behaviour of the xWCETT protocol given the dynamics inherent in the multi-hop $\mathrm{CRN}$ environment. The performance of the routing protocols was evaluated in terms of the average end-to-end latency, throughput, jitter, packet delivery ratio (PDR), and the normalised routing overhead (NRL). The results are based on the network size with sixty (60) SU nodes and six (6) PU nodes. In Figure 3, we present the average end-to-end latency results.

We can observe that the average xWCETT latency is lower compared with AODV and WCETT. The AODV and WCETT routing protocols had higher latency because they are not designed for the cognitive radio environment. The performance of AODV and WCETT degrades due to dynamics in the operating environment such as availability of spectrum. The sporadic availability of the spectrum causes WCETT and AODV to suffer increased delays. In Figure 4, we present the comparative average jitter results obtained by the three routing protocols. 
Table 1: CR-WMNs simulation parameters

\begin{tabular}{|l|l|}
\hline Parameter & Values \\
\hline Topography & $1000 \mathrm{~m} x 1000 \mathrm{~m}$ \\
SU nodes & 60 \\
PU nodes & 6 \\
SU radios (interfaces) & 2 \\
Mobility model & Random waypoint \\
Mobile client node speed & $1-10 \mathrm{~m} / \mathrm{s}$ \\
Transmission range & $150 \mathrm{~m}$ \\
MAC & IEEE $802.11 \mathrm{~b}$ MACCON \\
RF propagation model & Two Ray Ground Reflection \\
Antenna type & Omni-directional \\
Traffic type & CBR \\
Packet size & 1500 bytes \\
Data rate & $11 \mathrm{Mbps}$ \\
Routing protocols & AODV, WCETT, xWCETT \\
Simulation time & $300 \mathrm{~s}$ \\
\hline
\end{tabular}

The average end-to-end jitter performance results shown in Figure 4 indicate that the performance of xWCETT protocol is degraded by delay variations per packet delivery. The AODV and xWCETT obtained higher average jitter results as compared with the WCETT routing protocol. This can be attributed to the topology and spectrum dynamics. The WCETT routing protocol obtained the best jitter results in comparison with the xWCETT protocol. In xWCETT nodes maintains the channel availability table which is refreshed when the PU is detected. Subsequently, the ranking of the cost values in the channel availability table increases the network repair time which increased average jitter. The average end-to-end throughput and PDR results are presented in Figure 5 and 6 respectively. 


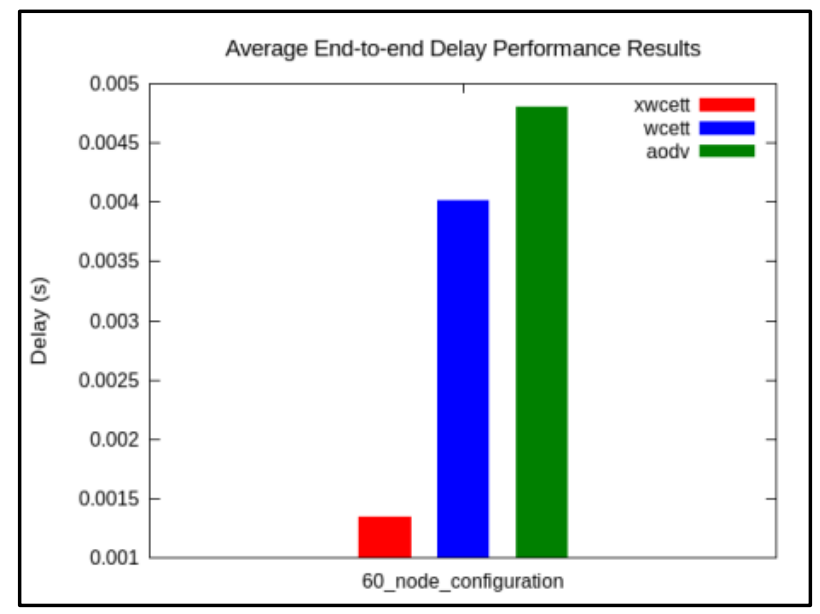

Figure 3: The average latency performance results

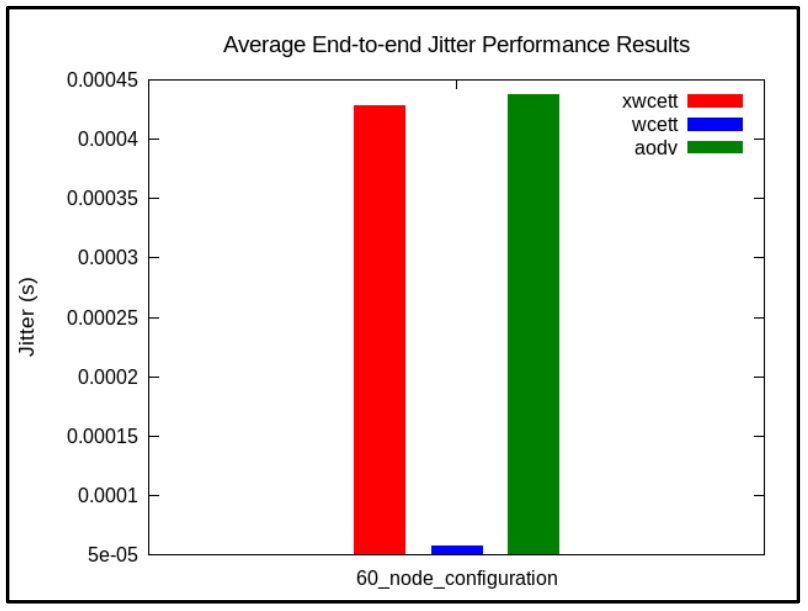

Figure 4: The average jitter performance results 


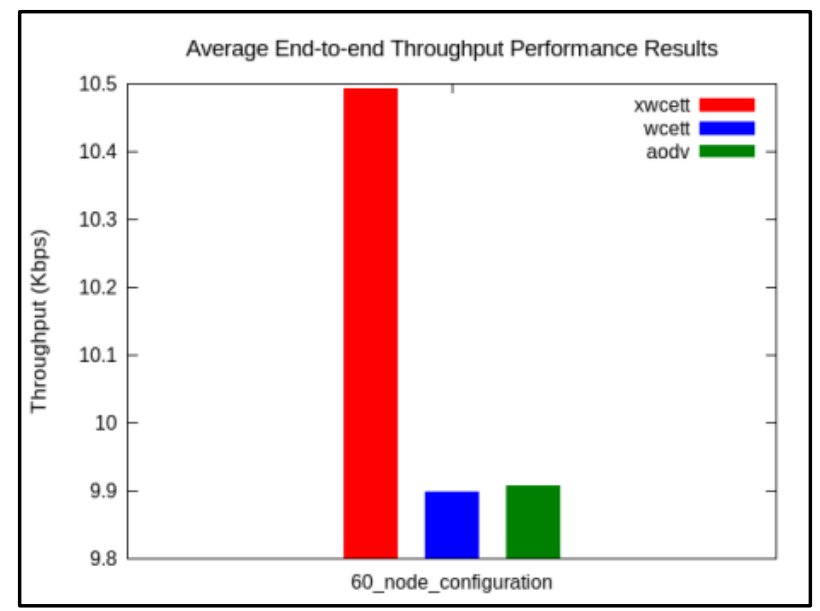

Figure 5: The average throughput performance results

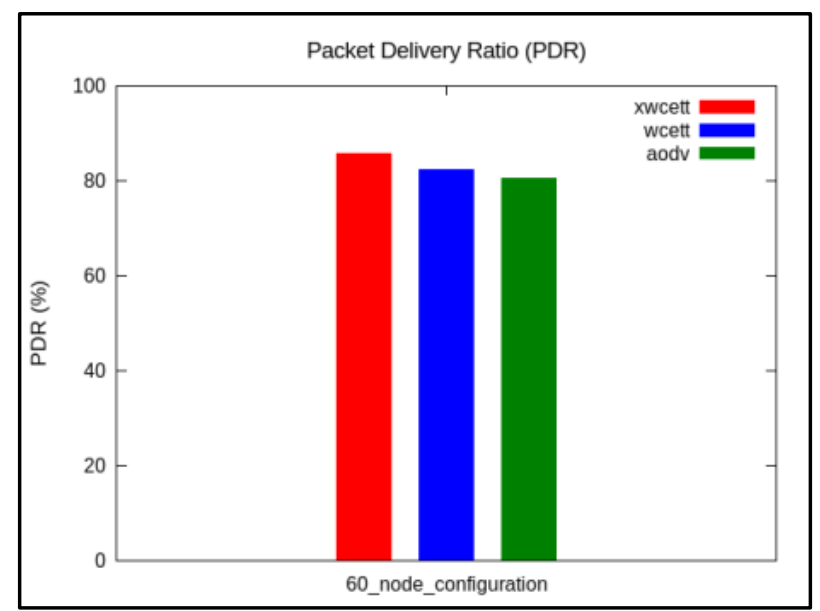

Figure 6: Packet delivery ratio results

The end-to-end throughput results depicted in Figure 5 demonstrate an unsteady performance in all three (3) routing protocols. The xWCETT protocol however obtained the highest achievable throughput than WCETT and the AODV protocols obtained the lower average throughput. The lower throughput indicates inefficient utilisation of the spectrum bandwidth as only fewer packets are successfully transmitted. This means that the WCETT and AODV routing protocols are unable to utilise the network resources efficiently as compared to the xWCETT routing protocol.

The PDR results show that xWCETT achieved the highest percentage of PDR. This shows that xWCETT protocol loses fewer packets compared to the WCETT and AODV protocols. Longer path repairs degrade PDR in the AODV and WCETT protocols. The xWCETT repairs routes faster. The NRL results are presented in Figure 7. 


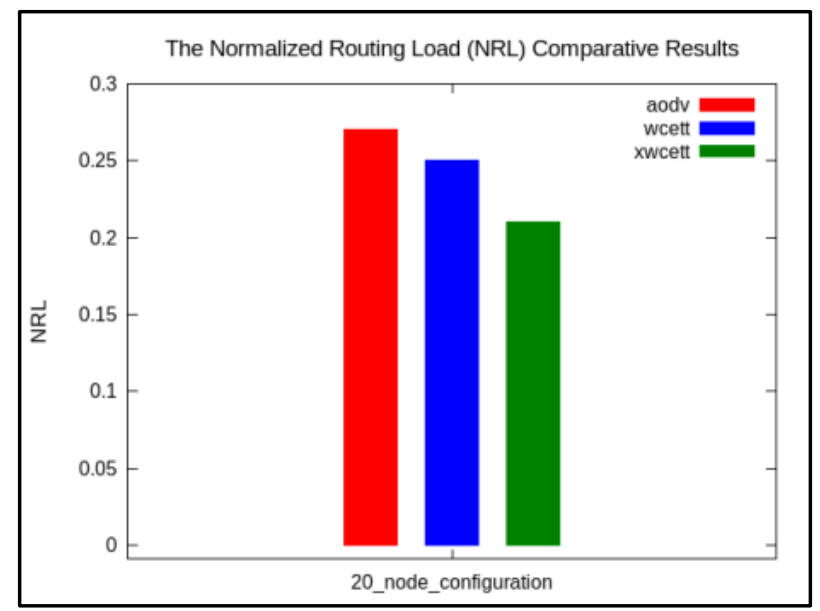

Figure 7: The NRL comparative results

In Figure 7, it was observed that the AODV protocol obtained the highest NRL because during the route establishment process, the AODV protocol broadcasts the routing packets on all available channels. The AODV protocol floods the network with routing packets, resulting in increased routing overhead. The higher routing overhead consumes more network resources which results in inefficient utilisation of bandwidth. The WCETT and xWCETT protocols compute the best path by considering the condition of the path. Thus, the WCETT and xWCETT provide better and more improved performance in terms of NRL because they are optimised for channel diversity. The xWCETT is the best performing protocol because it selects the best path.

We further analysed the performance of the routing protocols by increasing the number of SU network nodes to 100 . The total number of PU nodes was fixed at $10 \%$ of the total network nodes. Figure 8 presents the average latency results.

Figure 8 shows that latency is degraded by network size. Increasing the number of network nodes increases the average end-to-end latency. The same behaviour was observed in the case of jitter results, presented in Figure 9. The xWCETT protocol performed well in terms of the latency but its performance was poor in terms of the average jitter. The performance of xWCETT protocol is affected by the frequent updates and ordering of the channel availability table entries.

Figures 10 and 11 present the comparative throughput and PDR results respectively. The latency and jitter results showed that the performance of the CR-WMNs is degraded by the size of the network. This was also observed in the average throughput and PDR. This observation is clearly demonstrated by Figure 10, in which network performance degrades as the network size increases. The PDR results in Figure 11 show the gradual decrease in the average PDR as the network grows larger in size. The network size degrades more the real-time and/or delay sensitive constant bit rate (CBR) traffic. 


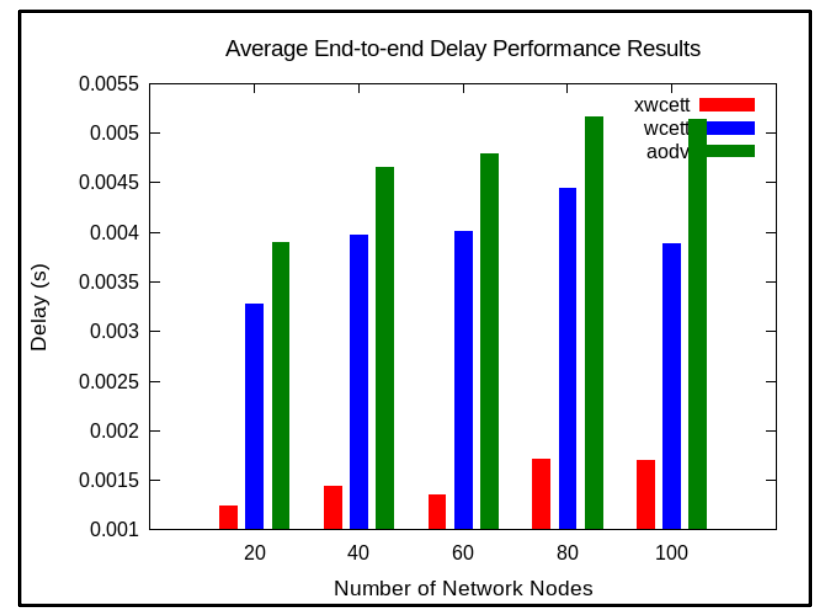

Figure 8: The average latency versus the number of SU nodes

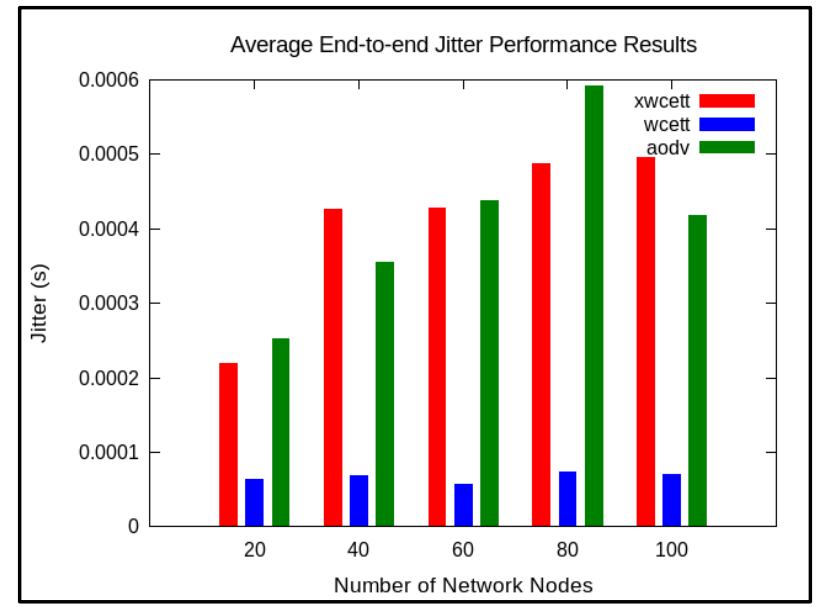

Figure 9: The average jitter versus the number of SU nodes 


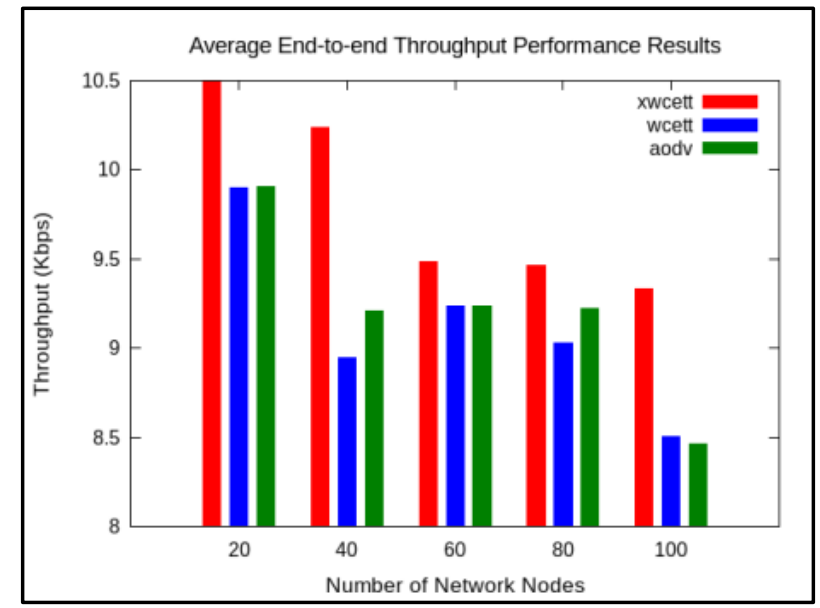

Figure 10: The average throughput versus the number of SU nodes

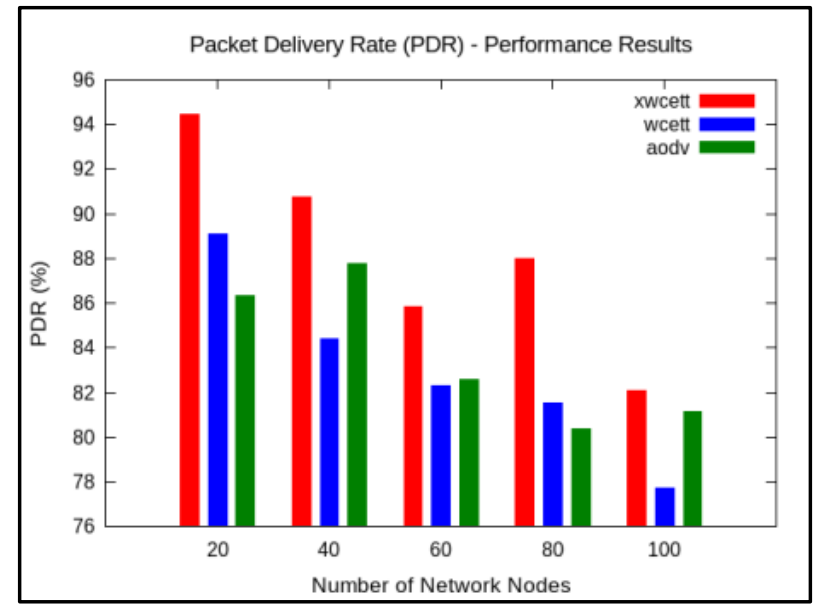

Figure 11: The average end-to-end PDR versus the number of SU nodes 
The comparative results in this study show that the overall performance of multi-hop CRN depends largely on the routing criteria implemented by the routing protocol. We further observed that the xWCETT protocol obtained significantly better results in comparison with the AODV and WCETT routing protocols. Its performance remained stable and consistent as compared to the AODV and WCETT routing protocols. However, the WCETT protocol achieved better jitter results because it broadcasts data packets over a set of all available channels. Furthermore, the xWCETT protocol frequently updates its channel availability and transmits only on a subset of available channels. The AODV routing protocol's performance was mostly unstable with high average latency and low throughput mainly because of it does not consider the conditions of channels.

\section{CONCLUSION}

This paper highlighted a number of routing issues and challenges encountered in CR-WMNs. The common challenge underpinning ineffective performance of CR-WMNs is attributed to the dynamic spectrum access and the dynamics in the re-configurability of network parameters. To address these challenges, we proposed a spectrum-aware, spectrum-agile, and interference-aware routing protocol the xWCETT. The xWCETT protocol integrates the AODV and the WCETT routing metrics into a new enhanced routing protocol. The xWCETT routing protocol is based on a distributed local spectrum knowledge whereby each SU in constructs the knowledge of its neighbourhood by sensing the spectrum for PU activities.

The xWCETT protocol was implemented in NS-2 simulators to evaluate its performance in comparison with the AODV and the WCETT protocols. The simulation results indicated that the xWCETT protocol outperformed the AODV and WCETT protocols in terms of latency, throughput, PDR, and the NRL. The WCETT protocol outperformed the xWCETT only in terms of the average jitter results. The xWCETT protocol maintained a steady and robust performance as the network size was increased from 20 to 100 nodes.

\section{ACKNOWLEDGEMENTS}

This work is based on the research supported in part by the National Research Foundation of South Africa for the grant Unique Grant No. 94077.

\section{References}

Akyildiz, I., Lee, W., \& Chowdhury, K. (2009). CRAHNs: Cognitive radio ad hoc networks. Ad hoc networks, 7(5), 810-836. https://doi.org/10.1016/j.adhoc.2009.01.001

Akyildiz, I., Lee, W., Vuran, M., \& Mohanty, S. (2006). NeXt generation/dynamic spectrum access/cognitive radio wireless networks: A survey. Computer networks, 50(13), 2127-2159. https://doi.org/10.1016/j.comnet.2006.05.001 
Akyildiz, I., Lo, B., \& Balakrishnan, R. (2011). Cooperative spectrum sensing in cognitive radio networks: A survey. Physical communication, 4(1), 40-62. https:// doi.org/10.1016/j. phycom.2010.12.003

Akyildiz, I., Wang, X., \& Wang, W. (2005). Wireless mesh networks: A survey. Computer networks, 47(4), 445-487. https://doi.org/10.1016/j.comnet.2004.12.001

An, B. (2014). A stability-based spectrum-aware routing protocol in mobile cognitive radio adhoc networks. In 2014 International Symposium on Computer, Consumer and Control (IS3C) (pp. 1014-1017). https://doi.org/10.1109/IS3C.2014.265

Bhatia, R. \& Li, L. (2007). Throughput optimization of wireless mesh networks with MIMO links. In INFOCOM 2007. 26th IEEE international conference on computer communications (pp. 23262330). https://doi.org/10.1109/INFCOM.2007.274

Bruno, R., Conti, M., \& Gregori, E. (2005). Mesh networks: Commodity multihop ad hoc networks. IEEE communications magazine, 43(3), 123-131. https://doi.org/10.1109/MCOM.2005. 1404606

Cacciapuoti, A., Calcagno, C., Caleffi, M., \& Paura, L. (2010). CAODV: Routing in mobile ad-hoc cognitive radio networks. In 2010 IFIP Wireless Days (WD) (pp. 1-5). https://doi.org/10. 1109/WD.2010.5657754

Che-Aron, Z., Abdalla, A., Abdullah, K., Hassan, W., \& Rahman, M. (2015). RACARP: A robustness aware routing protocol for cognitive radio ad hoc networks. Journal of Theoretical \& Applied Information Technology, 76(2), 246-257.

Che-aron, Z., Abdalla, A., Hassan, W., Abdullah, K., \& Rahman, M. (2014). E-D2CARP: A joint path and spectrum diversity based routing protocol with an optimized path selection for cognitive radio ad hoc networks. In 2014 IEEE 2nd International Symposium on Telecommunication Technologies (ISTT) (pp. 39-44). https://doi.org/10.1109/ISTT.2014.7238173

Cheng, H., Jiang, H., \& Zhuang, W. (2006). Distributed medium access control for wireless mesh networks. Wireless Communications and Mobile Computing, 6(6), 845-864. https://doi.org/ 10.1002/wcm.445

Doomari, S. \& Mirjalily, G. (2016). Interference aware shortest route selection in energy constrained cognitive radio ad-hoc networks. In International Conference of Signal Processing and Intelligent Systems (ICSPIS) (pp. 1-5). https://doi.org/10.1109/ICSPIS.2016.7869905

Fall, K. \& Varadhan, K. (2007). The network simulator (ns-2). Last accessed: 03 Dec 2017. Retrieved from http://www.isi.edu/nsnam/ns

Haykin, S. (2005). Cognitive radio: brain-empowered wireless communications. IEEE journal on selected areas in communications, 23(2), 201-220. https://doi.org/10.1109/JSAC.2004. 839380

Ho, Q., Tweed, D., \& Le-Ngoc, T. (2017). Requirements and regulations in the 5 ghz unlicensed spectrum. In Long term evolution in unlicensed bands (pp. 11-20). Springer. https://doi.org/ 10.1007/978-3-319-47346-8_2

Ishibashi, B. \& Boutaba, R. (2009). The symbiosis of cognitive radio and wireless mesh networks. In Guide to wireless mesh networks (pp. 471-496). Springer. https://doi.org/10.1007/978-184800-909-7_18 
Ji, Z. \& Liu, K. (2007). Cognitive radios for dynamic spectrum access-dynamic spectrum sharing: A game theoretical overview. IEEE Communications Magazine, 45(5). https://doi.org/10.1109/ MCOM.2007.358854

Lee, M., Zheng, J., Ko, Y., \& Shrestha, D. (2006a). Emerging standards for wireless mesh technology. IEEE Wireless Communications, 13(2), 56-62. https:// doi.org/10.1109/MWC.2006. 1632481

Lee, M., Zheng, J., Ko, Y., \& Shrestha, D. (2006b). Emerging standards for wireless mesh technology. IEEE Wireless Communications, 13(2), 56-63. https:// doi.org/10.1109/MWC.2006. 1632481

Meghanathan, N. \& Fanuel, M. (2015). A minimum channel switch routing protocol for cognitive radio ad hoc networks. In 12th International Conference on Information Technology-New Generations (ITNG), 2015 (pp. 280-285). https://doi.org/10.1109/ITNG.2015.51

Mitola, J. (1999). Cognitive radio: model-based competence for software radios (Doctoral dissertation, Tekniska högskolan i Stockholm. Institutionen för teleinformatik, Sweden).

Mitola, J. (2009). Cognitive radio architecture evolution. Proceedings of the IEEE, 97(4), 626-641. https://doi.org/10.1109/JPROC.2009.2013012

Noam, E. (1995). Taking the next step beyond spectrum auctions: Open spectrum access. IEEE Communications Magazine, 33(12), 66-73. https://doi.org/10.1109/35.476925

Pabst, R., Walke, B., Schultz, D., Herhold, P., Yanikomeroglu, H., Mukherjee, S., ... Aghvami, H. (2004). Relay-based deployment concepts for wireless and mobile broadband radio. IEEE Communications Magazine, 42(9), 80-89. https://doi.org/10.1109/MCOM.2004.1336724

Rahman, M., Caleffi, M., \& Paura, L. (2012). Joint path and spectrum diversity in cognitive radio ad-hoc networks. EURASIP Journal on Wireless Communications and Networking, 2012(1), 235. https://doi.org/10.1186/1687-1499-2012-235

Ramanathan, R. (2001). On the performance of ad hoc networks with beamforming antennas. In Proceedings of the 2nd ACM international symposium on Mobile ad hoc networking and computing (pp. 95-105). https://doi.org/10.1145/501426.501430

Sengupta, S. \& Subbalakshmi, K. (2013). Open research issues in multi-hop cognitive radio networks. IEEE Communications Magazine, 51 (4), 168-176. https://doi.org/10.1109/MCOM.2013. 6495776

Siraj, M. \& Bakar, K. (2012). Minimizing interference in wireless mesh networks based telemedicine system. Journal of computer science, 8(8), 1263-1271.

Sun, B., Zhang, J., Xie, W., Li, N., \& Xu, Y. (2012). A novel spectrum-aware routing protocol for multi-hop cognitive radio ad hoc networks. In 2012 International Conference on Wireless Communications \& Signal Processing (WCSP) (pp. 1-5). https://doi.org/10.1109/WCSP. 2012.6542813

Sutton, P., Doyle, L., \& Nolan, K. (2006). A reconfigurable platform for cognitive networks. In 1st International Conference on Cognitive Radio Oriented Wireless Networks and Communications, 2006 (pp. 1-5). https://doi.org/10.1109/CROWNCOM.2006.363467 
Tian, Z. \& Giannakis, G. (2006). A wavelet approach to wideband spectrum sensing for cognitive radios. In 1st International Conference on Cognitive Radio Oriented Wireless Networks and Communications, 2006 (pp. 1-5). https://doi.org/10.1109/CROWNCOM.2006.363459

Yadav, R. \& Mane, A. (2015). Lasar: spectrum aware routing protocol for cognitive radio wireless networks. In 2015 International Conference on Communication, Information \& Computing Technology (ICCICT) (pp. 1-6). https://doi.org/10.1109/ICCICT.2015.7045694

Zhang, D. \& Tian, Z. (2007). Spatial capacity of cognitive radio networks: Narrowband versus ultrawideband systems. In Wireless Communications and Networking Conference, 2007 (pp. 6-10). IEEE. https://doi.org/10.1109/WCNC.2007.7 